\title{
GILIINNA CARLGATI
}

\section{DANTE. IL "BREVE USO" DELL'NMORE}

\author{
"Non ti dorea gravar le penne in giuso, \\ ad aspettar più colpo, o pargoletta \\ o altra novità con sì breve uso". (Purg. 31, 58-60)
}

\begin{abstract}
"convenne, prima che questo nuovo amore fosse perfetto, molta battaglia intra lo pensiero del suo nutrimento e quello che li era contraro", "e per iscusare me de la varietade ne la quale parea me avere manco di fortezza, dirizzai la voce mia in quella parte onde procedeva la vittoria del nuovo pensiero" (Com!. II ii 3, 5).
\end{abstract}

"Norità", "breve uso"1, "varietade": ecco come l'Afrodite terrestre si connota per Dante. Né infernale né mortifera, ma puntuale e discontinua nel suo divino splendore, la dea si lascia catturare nell' "antica rete" per cui l'amore passa, in un punto solo, da estasi solitaria a spettacolo per il Vulcano di turno. È il suo "breve uso" quello che per Dante fonda la necessità, anzi, l'inevitabilità, di un superamento che, per quanto assicurato dalla vita, e dalla morte, ruole però essere "ragionato", e cioè portato alla parola. Ecco dove l'Afrodite terrestre si interseca, ineludibilmente, con la ragione: termine onnipresente in Dante, in un mosaico polisemico di cui generalmente solo le tessere più vistosamente difformi dal nostro uso corrente sono fatte oggetto di speciale considerazione. Non si riflette forse abbastanza sul fatto che la percezione del colore di ogni tessera, e quindi anche il suo uso, è determinato dall'insieme del mosaico. Così ad esempio, e senza pretendere di poter rianalizzare qui, per esteso, il campo semantico del termine, "ragione" in Dante non può non contenere sempre, in qualche modo, quel "ratio" che i latini avevano ritenuto adatto a tradurre i termini greci lógos, diánoia e talvolta nóesis "designando perciò sia il fondamento intellegibile, oggettivo, delle cose, sia la facoltà umana capace di cogliere questo stesso fondamento" (ED, 831) e che questa facoltà umana si esplica nel linguaggio. La lingua è infatti definita da Dante come "rationale signum et sensuale" (DI E, I iii 2), "operazione dell'anima razionale, dove la divina luce più espeditamente raggia" (Comr. IIl vii 8). Ora, il "non durabile" "effetto razionabile" che è il discorso degli uomini (cf. Par. 26, 
127-129), segnato dal "peccato", è, o dovrebbe essere, in continua tensione verso l'unità di una mitica pre-Babele, unità che recede infinitamente, come Paradiso 26 evidenzia, fino all'istante originario che il giardino dell'Eden rappresenta. Nel "frattempo" che è poi il tempo della storia, il solo tempo dell'umanità, il "provvisorio" rimedio all'anarchia delle lingue e degli appetiti individuali è la ragione, il comunicare degli uomini nella non ideale società che "subentra" alla solitudine edenica, cioè che da sempre ne ha preso il posto. La ragione è (era, sarà) una come è uno l' "intelletto" che procede da Dio e che è $\mathrm{Dio}^{2}$ : ma, distendendo se stessa nella storia, necessariamente si fa scrittura: "E con ciò sia cosa che in tutte queste operazioni sia equitade alcuna da conservare e iniquitade da fuggire (Y), trovata fu la ragione scritta, e per mostrarla e per comandarla. Onde dice Augustino: 'Se questa — cioè equitad- li uomini la conoscessero, e conosciuta servassero, la ragione scritta non sarebbe mestiere'; e però è scritto nel principio del Vecchio Digesto: 'La ragione scritta è arte di bene e d'equitade"' (Conv. IV ix 8). Dunque la ragione è anche, ovvero incessantemente diventa, parola scritta, grammatica formulata, dagli uomini e per gli uomini: essa sovrasta l'anarchia individuale per stabilire idealmente l'impossibile giustizia che non per niente è "di Dio".

È questa la ragione che "consiglia" Dante, e che lo consiglia precisamente a consegnare alla scrittura non già la passione, inconsegnabile in quanto tale, ma la meditazione sulla immagine che signoreggia, senza annichilare, il seguace d'amore. O almeno senza annichilarne l'ingegno di scrittore: "E arvegna che la sua imagine, la quale continuatamente meco stava, fosse baldanza d'Amore a segnoreggiare me, tuttavia era di sì nobilissima vertù, che nulla volta sofferse che Amore mi reggesse senza lo fedele consiglio de la ragione in quelle cose là ove cotale consiglio fosse utile a udire" ( $\mathrm{N}$ ii 9). Che questo intervento della ragione non sia da vedere sullo sfondo di una "colpevole passione" di stampo ottocentesco, lo conferma $I \mathrm{~N}$ iv 2, dove la ragione non fa che consigliare a Dante, come del resto voleva il libro di Gualtieri, di non rivelare l'identità delle sue frequentazioni femminili (ammesso, e tutt'altro che concesso, che la fanciulla che diventerà "miracolo" rientri in questo novero a pieno titolo): "Ed io, accorgendomi del malvagio domandare che mi faceano, per la volontade d'Amore, lo quale mi comandava secondo lo consiglio de la ragione, rispondea loro che Amore era quelli che così m'avea governato. Dicea d'Amore, però che io portava nel viso tante de le sue insegne, che questo non si potea ricorrire. E quando mi domandavano "Per cui t'ha così distrutto questo Amore?', ed io sorridendo li guardava, e nulla dicea loro". Dore, così almeno pare dica il testo, la ragione, in un gesto che sarebbe 
sbagliato qualificare come cinico, si preoccupa esclusivamente di mantenere aperta la conversazione intorno ad amore, mettendola contemporaneamente al riparo dalle invidie, dalle ripicche e dalle gelosie che la renderebbero "ingiusta".

La conversazione intorno ad $A$ more è, o dovrebbe essere per l'implacabile spinta ad unum che è caratteristica della mente di Dante, incentrata intorno a una sola amata, la prima intatta, e perciò inattingibile, fanciulla. Cosi vuole "ragione", la ragione che pensa e che parla, c disobbedire al suo mandato è peggio, forse anche più "doloroso", del non essere corrisposti: "Allora cominciai a pensare di lei; e ricordandomi di lei secondo l'ordine del tempo passato, lo mio cuore cominciò dolorosamente a pentere de lo desiderio a cui sì vilmente s'area lasciato possedere alquanti die contra la costanzia de la ragione: e discacciato questo cotale malvagio desiderio, sì si rivolsero tutti li miei pensamenti a la loro gentilissima Beatrice" (I N xxxix 2). È qui che si situa il "dissidio" con Cavalcanti. Guido non vede come dall'amore carnale possa nascere ragione, orvero come amore e ragione idealmente si coniughino nella parola, amore che detta e ragione che scrive: l'amore e l'intelletto, l'uno mortale, l'altro eterno, sono radicalmente dissimili, nessuna scintilla divina è capace di penetrare l'oscurità del corpo: l'intelletto "non ha diletto - ma consideranza; sì che non pote largir simiglianza" (Donna me prega, 26-27). Nulla di più lontano dal pensiero di quella Afrodite celeste, anima del mondo, distribuito in mille rivoli per tutta la plurisecolare cultura neoplatonica, che funge da intertesto subliminale nell'immaginazione dantesca: nessuna "figura" per Guido, nessuna "trinità", nessuna Beatrice. Eppure Guido dice una verità che Dante non potrà superare mai, se non nella sospensione della catacresi poetica: ché il "breve uso"che è l'amore carnale, pur se penetrato dal dio, anzi, proprio perché penetrato dal dio, mai sarà altro che momentaneo affondare nella u-topia.

Amore e ragione si travasano l'uno nell'altra, amore può entrare neila storia umana solo facendosi parola, la parola umana può solo nascere da amore, che chiama l'uomo a sè stesso attraverso gli occhi dell'altro. La I 'ita nuora non è "superata" dalla Commedia, la donna gentile non è la "sgualdrinella" da scartare a favore di una (possibile) Bice Portinari educata e vereconda, dal cui virtuoso riserbo Dante sarebbe (stato) "rimesso al suo posto", e le sue arances ricondotte entro l'ambito di una borghese ragionevolezza. Un maturo componimento quale Io sono stato con amore insieme non è il cedimento a una visione dell'amore che sarà confutata nella Commedia, non è il prodotto di una momentanea distrazione, ma è, appunto, la matura meditazione di un uomo che accetta la precarietà dell'amore 
carnale e la sua scandalosa incongruenza, pari soltanto all'incongruenza, in un mondo divino, del male e della morte. Colui che "sprieme" (v. 1) contro Amore ragione o virtù, colui, cioè, che è consapevole che amore accade "prima" della conversazione sociale, sa benissimo che questa consapevolezza è vana, come è vano suonare le campane per far cessare un temporale, "credendo far colà dove si tona/ esser le guerre de' vapori sceme". La quale similitudine apre per così dire verso l'alto l'orizzonte solo apparentemente tutto terreno del sonetto (ma c'era già quella "circulazion del sol", al v. 2, che allarga al cosmo l'orizzonte privato del poeta): "colà dove si tona" è già palestra d'amore, centro del cerchio che in sé racchiude - $\mathrm{e}$ come non potrebbe? - perfino il "libero arbitrio". Dante non sta affatto consigliando l'amico Cino a piantare una donna per l'altra, si limita a constatare il breve uso dell'amore: "E qual che sia 'l piacer ch'ora n'addestra,/ seguitar si convien, se l’altro è stanco": desiderio chiama desiderio, "Amor ch'a nullo amato amar perdona". Così "conviene", così è inevitabile che sia, così è misteriosamente voluto "colà dove si puote ciò che si vuole": "più non dimandare". Stupisce che si continui a vedere Dante come il perbenista che è convinto di essere diverso dagli altri o come il moralista che primariamente si dedica a castigare i costumi di una società corrotta: il "subiectum" dell'opera dantesca tutta, è "homo", "prout merendo et demerendo", il quale, comportandosi bene o male "per arbitrii libertatem", "iustitie premiandi et puniendi obnoxius est", si colloca nell'orizzonte illimitato e imperscrutabile della giustizia: divina, e cioè "rimandata" alla fine del tempo. Le parole di Dante, i destinatari, i contesti cambiano: il pensiero "cammina", si approfondisce, si cerca. Sulla brevità e divinità dell'amore carnale Dante non si corregge, mai.

\section{L'“orto di ragione"}

La canzone Doglia mi reca è una meditazione appassionata, forse colorata da una privata delusione, sul tema del rapporto tra giustizia e amore, e ovviamente dettata da quel desiderio di rifacimento di immagine e di conquista di autorevolezza cui il poeta è costretto a sottoporsi nei primi anni del suo esilio. Una canzone, dunque, squisitamente di ragione: della ragione che regge, o dovrebbe reggere, l'umano conversare, in vista di una giustizia fieramente voluta, mai conquistata né conquistabile prima che giunga "colei che ne pareggia" (v. 7), e pensabile "dopo" solo da un" "alta fantasia" che spera. $A$ differenza delle grandi canzoni sull'amore, Doglia mi reca ha una destinataria precisa, se pure non più da noi identificabile: una fiorentina, una Giovanna, una nobildonna. Che Dante sembra dispiacersi di veder circondata da "male bestie" che "con tardare", con "vana vista", con "sem- 
bianza trista" (гv. 119-120) trasformano "il donare" "in render" (v: 121), come sa chi paya tale supponenza (v: 122) a un prezzo cosi alto che in seguito "non li pare amaro" "I negar", il rifiutare il falso dono proffertogli (v: 125). Gesto non propriamente "politico", a considerare l'indigenza dell'esule, e parlo non del rifiuto, ma dell'invio della geremiade a persona che si immagina intima del mal disposto benefattore. E chissà che la canzone non fosse stata almeno subliminalmente pensata come un ultimo invito certamente destinato a fallire! - alla donna, bella e saggia, perché ricatti l'uomo negandoglisi. "Dico che bel disdegno/ sarebbe in donna, di ragion laudato,/ partir beltà da sé per suo commiato" (rา. 19-21)33. Si, perché la bellezza che amore per "decreto antico" (v. 9) conferisce a donna (cf. v. 7) è offerta all'uomo, attraverso il dono dello sguardo, perché questi ci mediti, ne ragioni, ne comprenda l'origine divina. Amore non riconosciuto come tale è insieme causa e conseguenza dell'ingiustizia. Già Andrea Cappellano, che Dante supera sì, ma solo in profondità teologica, redeva la giustizia come conditio sine qua non dell'amore: "Norum probitas acquirit amorem in morum probitate fulgentem", "Prodezza di costumi cerca amore, nella prodezza di costumi risplendente"t; già il De amore raccomandava che, qualora "costretta" da amore, solo ai giusti la donna si concedesse: "solis bonis vestrum esse largiendum amorem", "solo alli buoni dare dovete il vostro amore" (De amore I, 6).

Ma l'ingiustizia è la condizione dell'uomo errante, la giustizia non è di questo mondo. Il grido del poeta rimane senza risposta: "Morte, che fai? Che fai, fera Fortuna, che non solvete", che non distribuite, "quel che non si spende?" (vт. 90-91. "Non so" (เ. 93), scrive il poeta: "tal cerchio ne cinge" (v. 93), siamo circondati dal mistero. Né Dante correggerà mai questa sua conclusione, che ha il tragico splendore della verità: il Agiudicio di costei", della Fortuna, "è occulto come in erba l'angue. / V'ostro saver non ha contasto a lei: / questa provede, giudica e persegue/ suo regno come il loro li altri dei" (Inf. 7, 83-87). Al credente non viene davtrero nessun privilegio, né potrebbe essere diversamente: il "sommo Giove", che è forse alluso anche nel "colà dove si tona" del sonetto Io sono stato con amore insieme, tiene, di divina necessità, "li giusti occhi [suoi] rivolti altrove"; il bene, se e quando esso si dà ("alcun bene"), è "in tutto de l'accorger nostro scisso" (Purg. 6, 118-123). E la ragione? Muta anch'essa: la storia, la legge, la lingua del mondo che ha "perso" la giustizia originaria, non sa "castigare" l'ingiustizia, non sa correggerla. E in un mondo "fuor d'orto di ragione" (v. 147), in cui ragione non parla, amore non "passa", lo spirito muore. Eppure l'amore carnale non può non risiedere fuori di quest'orto, come tutta la tradizione non si stanca di ripetere. 
Seguiamo per un momento gli andirivieni logici di san Tommaso, all'articolo 34 della Summa (1a 2ae). "Videtur quod omnis delectatio sit mala", sembra che ogni piacere sia cattivo, poiché "il piacere corrompe la prudenza e impedisce l'uso della ragione, e tanto più quanto più grandi sono i piaceri. Per questo 'nei piaceri venerei', che sono i più grandi, 'è impossibile comprendere alcunché", dove Tommaso cita Aristotele. "Sed contra", il salmo 36 della Vulgata dice "Delectare in Domino" ("et dabit tibi petitiones cordis tui", continua il versetto, 4, che Tommaso non cita per intero). Se "la divina autorità dice cosi — continua la Stmmma- "videtur quod non omnis delectatio sit mala", sembra che non tutti i piaceri siano cattivi. E allora? Ecco la risposta che, senza concludere, mette fine alla dimostrazione: "i piaceri che fanno parte della ragione non impediscono la stessa né corrompono la prudenza"; l'opposto vale per i "piaceri estranei" alla ragione, "quali i piaceri corporali". Infatti questi piaceri extranei "impediscono l'uso della ragione o perché sono diretti verso qualcosa che ripugna alla ragione, il che rende il piacere moralmente riprovevole, o perché, e qui veniamo all'amore carnale, accadono "secumdum quandam ligationem rationis", per così dire a ragione neutralizzata, così come avviene "in concubitu coniugali". Sebbene in esso ci sia qualcosa che "alla ragione non contraddice", l'amore carnale impedisce l'uso della ragione "propter corporalem transmutationem adjunctam", per via della trasmutazione corporea che vi si accompagna". In questo non c'è "malizia morale", come non può essercene nel sonno, dove pure la ragione è "legata": infatti "la ragione stessa esige che l'uso della ragione sia a volte interrotto". E tuttavia il "ligamentum rationis" che è intrinseco al piacere dell"atto coniugale", pur non comportando "malizia morale", proviene da una certa malizia "ex quadam morali malitia", e cioè dal peccato del primogenitore. Infatti "hoc", le cose, non stavano così nello stato d'“innocenza" originario. Ecco dove si ferma la spiegazione di san Tommaso: una rolta chiarita l'estrancità del piacere alla ragione, l'intrinseca contradditorietà dell'amore carnale risalta tragicamente in un mondo il cui guasto viene estromesso in quella che è in realtà un" "origine" fuori del mondo. Dove la "colpa originale" diventa scatto iniziale che inaugura il mondo e la sua tragica (co)scienza del bene e del male. Sulla intrinseca divinità dell'amore carnale, del piacere della carne, Tommaso, come è ovvio, non potrebbe dilungarsi. E del resto ogni filosofico dilungarsi, come dimostra la "dimostrazione" tomistica, è per definizione impossibile: dove viene a spegnersi la logica, dovrebbe nascere la metafora, a cui Tommaso, legato sto per dire professionalmente alla metafora biblica, fa più fatica a ricorrere di altri filosofi (si pensi a Platone o a Plotino). 
La riflessione poetica dantesca lascia intatta, e anzi ulteriormente divarica, "prima" di colmarla col "ragionare" della poesia, la spaccatura tra cros e storia. Molto significativa è l'ellissi, poco sottolineata dai commentatori, per cui, nel famoso passo di Purgatorio 16, la condotta umana viene come compressa tra piacere e legge, senza l'ovvio intermediario della ragione intesa come facoltà raziocinante individuale. "L'anima semplicetta che sa nulla" (v. 89), "a guisa di fanciulla che piangendo e ridendo pargoleggia" (rv. 86-87), "volentier torna a ciò che la trastulla" (v. 90): dunque l'uomo è essere di desiderio, e i termini che lo descrivono sono gli stessi che Dante usa in riferimento al desiderio amoroso. Poteva accennare, il poeta, al "pappo e 'l dindi" (Purg. 9, 105) del bambino, e invece ci mette davanti agli occhi le pargolette amorose, quelle che insieme con l'uomo portano il giogo d'amore e sanno "come sotto di lui si ride e geme" (Io sono stato con amore insieme, v. 4). Il pargoleggiare arviene "piangendo e ridendo": dunque il "piangere" è inseparabile dal desiderio; anche Dante sa, e non per averlo appreso dall'amico Guido, che amore è "morte". Eppure l'anima pargoletta, attraverso le lacrime, torna instancabilmente a ciò che la trastulla, che è la lietezza, divina, dell'amore, cosi come vuole il suo "lieto fattore" (v. 89). Essa corre dietro "picciol bene", ma vi si "inganna": vorrebbe restarvi ogni volta, recidiva ignara della breve durata, del "breve uso", di questo, come di ogni altro "bene". Salvo che non intervenga "guida o fren", metafora che ognuno sarebbe tentato di risolvere immediatamente in "ragione", nel senso corrente, come facoltà di cui Dio ha dotato l'uomo creandolo, destinata a svilupparsi con il maturare della persona. E inrece no, l" "onde" che si attacca a "se guida o fren non torce suo amore" (v. 93) introduce "convenne legge per fren porre" (v. 94), come dire che "prima" viene il consorzio umano, "poi" la ragione che ne è lo strumento. E Dante rincara la dose: "convenne rege aver, che discernesse/ de la vera cittade almen la torre" (vr. 95-96). L'anima pargoletta che sa nulla — ma che, per volere di Dio, si diletta "in Dio" — non conosce la "ragione" se non attraverso la conversazione degli uomini. Il Monarchia, mentre nomina quel che qui non compariva, cioè il peccato originale, conferma tuttavia che il primo pensiero di Dante corre alla società e al suo darsi una legge: "si homo stetisset in statu innocentie in quo a Deo factus est, talibus directivis non indiguisset: sunt ergo huiusmodi regimina remedia contra infirmitatem peccati" (III iv 14-15). In Purgatorio 16 si insiste che "la cagion che "I mondo ha fatto reo" non è "natura che 'n roi sia corrotta", bensi "mala condotta", e cioè cattivi governanti di una socictà peraltro perennemente malgovernata. "Cavalcatore de la umana rolontade", dice infatti il Commino, è "lo Imperadore" (IV ix 8-10), si direbbe quasi 
senza quell'intermediario che è il personale giudizio della ragione intesa come facoltà. In assenza del principe, l'anarchia dei desideri è -O dovrebbe essere - ridotta ad unità da quel "gratioso lumine rationis"che nel contesto del De inlgari (I xviii 5) sembra dover coincidere con la lingua. Ragione e storia nascono insieme, e insieme ad esse nasce (come potrebbe essere altrimenti?) la lingua storica degli uomini: nel Paradiso Terrestre, al brevissimo soggiorno di Adamo - sei ore!- basta una lingua in cui si dice l'indicibile "I" di un Dio senza nome (cf. Paradiso 26, 134).

\section{"Se fosse amico il re de l'universo"}

La principale prova del "superamento" della poetica e della filosofia dello stil nuovo in direzione di un amore pacificamente ragionevole sarebbe, per molti, la "condanna" del peccato di Francesca, condanna volta a volta accompagnata dal disprezzo, aguzzata dall'ironia, mitigata dalla nostalgia, addolcita dalla "pietà". Diciamo subito che impostare una lettura di Inferno 5 in termini di condanna significa costringere la straripante originalità poetica della Commedia entro gli argini di un moralismo che sicuramente non ne esprime la grandezza. Sull'amore Dante medita da sempre, da quel primo sonetto della I ita nuova che ancora traveste di "sogno" l'irriducibile dilemma di un eros che ride e piange, che chiama e risponde a gentilezza pur suscitando contemporaneamente "orrore". E che quindi si configura già come problema filosofico, nell'imprescindibile opposizione tra la "stasi", la permanenza temporale dell'umana conversazione e l'e-stasi fuori dal tempo del silenzio della carne. Il canto V dell'Inferno rappresenta forse il più drammatico momento di questa meditazione, il momento in cui Dante "risponde" ai propri dubbi sulla contradditorietà intrinseca dell'eros, e vi risponde non già condannando, ma innalzando la contraddizione ad insolubile dilemma teologico.

Il pittoresco aspetto bestiale di Minosse ha fatto a volte dimenticare quella che, per tutti i commentatori medievali, è la sua caratteristica essenziale: Minosse è innanzi tutto giudice, personificazione di una giustizia divina che è tale solo se surrogata dalla parola poetica. Per Jacopo Alighieri le due principali istanze che si esprimono nella figura di Minosse sono giustizia e coscienza, dore coscienza, come ragione, potremmo commentare noi, non è altro che l'interiorizzazione di quella "legge" che è la parola collettiva della società: "perciò cotale coscienza, nominata Minos, figurativamente in questo secondo grado principalmente si pone... a similitudine d'alcun re di Creta, nominato Ninos, il quale anticamente fu di tanto giusto giudizio abituato, che per ciascun pagano si credea che nello inferno finalmente giudicator divenisse" (Dartmouth Dante Project, Inf. 5, 4- 
6). Il Lana scrive che "questo Minos moralmente parlando significa giustizia ed è uno punitore di vizii" (ibid). Per l'()ttimo "Ninos è in figura di uno discreto e giusto giudice, si come fu discreto e giusto Minos, Re dell'isola di Creti, il cui figliuolo fu Minotauro" (ibid). "Conoscitor de le peccata", dunque, e in particolare, data la contiguità immediata coi "carnali", conoscitore di "quelle" peccata: ma le parole non connotano affatto quella "rozza carnalità" che caratterizzerebbe l'apertura del canto (Barolini, 37). La carne non conosce e non potrebbe giudicare. Semmai nella figura antonomastica che lo definisce sarebbe da cogliere un'allusione alle peccata di cui il destino ha voluto che Ninosse fosse testimone, dalle "imbestiate schegge" della moglie Pasifae (cf. Purg. 26, 87), alla spietatezza e perfidia della "noverca" Fedra (cf. Par: 17, 47). Forse dorremmo anche noi imparare dai commentatori medievali a non fare gran caso delle caratteristiche bestiali di Minosse — caratteristiche che poi si riducono al "ringhiare" e alla famosa coda - visto che esse non fanno altro che riecheggiare un' 1 conografia diffusa. Molto più marcati sono comunque $\mathrm{i}$ segni che introducono inequirocabilmente il tema della giustizia, per cui il "ringhiare" è come neutralizzato dalle funzioni del personaggio simbolo, che sono l'esame, il giudizio, la condanna: "essamina le colpe ne l'intrata;/ giudica e manda secondo ch'arringhia" (rv. 5-6). Le anime "mal nate", quelle che anziché edificare la società la distruggono, "confessano" assai poco bestialmente a Minosse, il quale "vede" qual è il loro peccato (rv: 9-10); esse "vanno a vicenda" (l'espressione suggerisce una certa civiltà di comportamento - al "giudizio", che è di nuoro parola nobile, appartenente alla sfera della razionalità. E le anime "dicono e odono", si direbbe quasi cortesemente, certo dignitosamente. Né le parole con cui Minosse, "a cui fallar non lece" (Inf. 29, 120) lascia "l'atto di cotanto offizio" (v. 17) appartengono a un registro "bestiale": "guarda", grida il giudice infernale, "non t'inganni l'ampiezza dell'entrare", mentre la risposta di Virgilio, al di là della preziosità stilistica della formula, adombra addirittura quello che sarà il tema centale del canto, ciò̀ l'articolazione inspiegabile - "e più non dimandare" - tra giustizia ed eros, tra storia ed eternità: "vuolsi così colà dove si puote ciò che si ruole".

E alla convivenza umana che il poeta guarda, con angosciata perplessità di intelletto - la "pietà" su cui tanto si è scritto— meditando sugli sconvolgimenti che nella società umana ha provocato e provoca la passione amorosa. Non si spiega altrimenti il fatto, forse non sufficientemente sottolineato dai commentatori, che tra "i peccator carnali,/ che la ragion sommettono al talento" (เт. 38-39) vengano poi nominate, o prescelte a protagoniste, solo "ombre" "ch'amor di nostra vita dipartille" (เ. 69), 
anime che, in diversa misura e con conseguenze più o meno gravi, tinsero "il mondo di sanguigno" (v. 90). Di Semiramide non si dice, né peraltro si esclude, che la sua morte fu riolenta: ma la favolosa regina, "imperadrice di molte favelle", è ben significativamente, e con possente rilievo, situata in testa alla fila di queste "genti", come colei che intervenne su quella legge che è la radice stessa della società, modificandola a capriccio: "che libito fe" licito in sua legge" (v. 56). Il "peccator carnale" di Dante non è davvero il fedele post-tridentino che si accusa, per dirla con Meneghello, di "atinpuri", ma è prima e sopra tutto colui che, tradendo il patto sociale, vien meno alle sue responsabilità di fronte alla storia. La perifrasi che connota Didone, colei che "ruppe fede al cener di Sicheo" (v: 62), è più omaggio a $V$ irgilio che insostituibile tessera del drammatico mosaico che si viene formando sotto i nostri occhi, unico riferimento a una dubbia "infedeltà coniugale" in un canto che sulla infedeltà coniugale parrebbe dorersi incentrare, mentre l'altra perifrasi, "colei che s'ancise amorosa", si situa in significativo hysteron proteron. In realtà Didone sembra solo chiamata in causa perchè Dante, insieme con Virgilio, la considera parzialmente responsabile, surrogando l'intoccabile Enea, delle incalcolabili conseguenze storiche che arrebbe aruto una rinuncia dell'eroe alla sua missione. Come del resto è provato dal passaggio del Convivio che illustra come "l'appetito [convenga] essere cavalcato da la ragione": "E quanto raffrenare fu quello, quando, avendo riceruto da Dido tanto di piacere quanto di sotto nel settimo trattato si dicerà, e usando con essa tanto di dilettazione, elli si partio, per seguire onesta e laudabile via e fruttuosa, come nel quarto de l'Eneida scritto è!” (IV xxvi 6;8). La stessa prospettiva storica vale per Elena e Paride e Achille, a causa dei quali in un modo o nell'altro "tanto reo/ tempo si volse" (เт. 64-65), e per Tristano, responsabile di un delitto che è ben più grave del peccato della carne, e per di più compiuto proprio da chi per la sua posizione regale dorrebbe incarnare la legge stessa della società. "Caina attende chi a vita ci spense": queste parole non servono solo a identificare il perpetratore del delitto, nè a caratterizzare quel certo "riserbo e velo" che sarebbe "tipico del parlare di Francesca" (Chiaracci Leonardi, I, 58). La quale, non contro ma insieme al poeta, senza pentimento ma anche senza odio, sottolinea così l'ultima conseguenza del proprio cedimento, il delitto fratricida in primo luogo, uxoricida forse secondariamente, che è gravissima infrazione al patto sociale. Ecco ciò che "condanna" Dante, o piuttosto ciò di cui a Dante importa che si prenda atto: che cioè "ragione", "legge", "storia", in un piano divino davrero incomprensibile, non appartengono all'ordine dell'eros, che è anch'esso di ordine divino.

Il poeta non rinnega, non condanna, non si distacca dalla dottrina d'amore così solennemente ribadita per bocca di Francesca e tutt'altro che 
contrastata dal "pellegrino", come non si distaccherà dalla passione civile di Farinata, dalla sacrosanta indignazione di Pier della V'igna nei confronti della "meretrice che mai da l'ospizio/ di Cesare non torse li occhi putti" (Inf. 13, 64-65), dall'ambizione intellettuale e pedagogica di Brunetto. İ vero, rimane sempre vero per Dante, che per sempre obbedirà al dettato d'amore, che amore "al cor gentil ratto s'apprende": non l'avera appena ribadito, per esempio, in Doglia mi reca? Ii non i, yuella dell'amante senza nome, "bella persona", cioè il contrario della "mala bestia" di cui si indigna il poeta nella "canzone della liberalita"? Come può Dante condannare, nonché il "padre [suo] e de li altri [suoi] miglior che mai/ rime d'amor usar dolci e leggiadre" (Purg. 26, 97-99), anche il se stesso che per stabilire la divinita dell'amore carnale sta ancora lavorando, adesso, a limare la figura di Beatrice? Cosi come non può non avere il sapore di un'eterna verità, per Dante come per noi, che "amore" "a nullo amato anar perdon[i]": non appartiene forse alla definizione stessa di desiderio di essere, molto prima che brama di soddisfazione sensuale, desiderio del desiderio dell'amato? Quella che si esprime in queste parole non è affatto una "concezione fatalistica" dell'amore (Chiavacci Leonardi, I, I57) che Dante condannerebbe dall'alto di una sua pretesa superiorità morale; se di fatalismo di tratta, è il fatalismo di chi rispetta il mistero del mondo: "State contenti, umana gente, al quicl" (Purg. 3, 37). In nessuna sua parte il testo si piega in senso volgare o bestiale, gli amanti non sono presentati, come da quasi tutti i lettori è stato sempre riconosciuto, in una cruda luce di sensualità: qui si parla di "vero amore", per noi come Dante. Se condanna c'è, essa ra cercata, metatestualmente, nella finzione su cui la finzione del poeta si regge, ma allora non si tratta tanto di condanna quanto di denuncia: io qui presente, Dante poeta, vi racconto alla maniera infernale una storia di cui non sono testimone bensi inventore, sapendo benissimo che gli effetti su di roi potrebbero essere analoghi a quelli che nella mia storia si rappresentano. Ed ecco la presa di distanza, la denuncia che si esprime attraverso la finzione della lettura nella lettura. Il racconto, nella sua sfacciata fittività, e nel suo potenziale di evangelico scandalo, non può prodursi senza un qualche avvertimento, se è vero che per Dante l'unica legittima scrittura è la scrittura "di Dio", intorno a Dio, la scrittura che interminabilmente si affina verso il divino e verso la morte.

Alla zona del divino appartiene l'insistita "pietà" del poeta, la sua muta, angosciata, irrisolvibile perplessita. "S'altri nol niega" (v. 8I), fa dire il poeta al se stesso personaggio, e queste parole trotano una eco in quel- 
lo straordinario "se fosse amico il re dell'universo" (v. 91) con cui Francesca esprime la sua simpatia verso l'"animal grazioso e benigno" che si rivolge a lei. Io credo che queste parole vadano accostate a quelle con cui Dante, stavolta nella primissima persona dell'uomo e del poeta, si rivolge a Dio chiedendogli ragione del male che devasta il mondo. Parole le cui tremende implicazioni sono denunciate da quella "captatio misericordiae" del "se licito m'è": "sono li giusti occhi tuoi rivolti altrove?" (Purg. 6, 118120). Dante non capisce, qui come in Inferno 5. Da questa metafisica incomprensione, e non da una assurda pietà dello scrittore per la propria invenzione, deriva l'immenso pathos del canto. Na come? Come può essere che lo stesso amore carnale, intuito ed espresso come divino dalla I'ita nuora al Convinio e oltre, sia poi causa di tanto "mal perverso" (v. 93)? "Chi" "ha voluto" questo stato di cose? Dove, come, quando, può darsi che questo amore "sia", e sia, come deve essere e come di fatto è, fonte suprema di salvezza?

\section{"e 'l pensamento in sogno trasmutai" (Purg. 18, 145)}

Il paradiso terrestre degli ultimi canti del Purgatorio dorrebbe essere nell'intenzione del poeta avreramento del sogno di Lia. Nella realtà del testo esso si colora invece della caratteristica utopica del sogno: tra coloro "che forse in Parnaso esto loco sognaro" $(28,141)$, resta ineludibilmente inscritto, "sesto tra cotanto senno" (Inf. 4, 102), anche il poeta che, in una pletora di riferimenti a testi "profani" — tra cui spicca solitario il Delectasti biblico- mette in scena la definitiva aporia filosofica e teologica dell'eros. Il muro di fuoco, le "flammantia moenia mundi" già di Lucrezio (De rerum natura, I 73), che il poeta fa trapassare al se stesso protagonista, non rappresenta soltanto la purificazione dal peccato della carne, ma è il crogiolo finale in cui la vita si distrugge per passare oltre se stessa, orvero la figura di un eros che variamente si stende lungo l'arco della vita, coprendo il mondo tutto nella sua tragica vicenda fino al momento in cui questo, in framme, precipita in altro. L'alta fantasia del poeta crea, tra mondo ed extramondo, tra storia ed eternità, un adynaton assoluto, un non-luogo di felicità che non è raggiungibile mai, nemmeno nella fede che pure in qualche modo fa da impalcatura alla farola del riaggio attraverso i tre regni. Come ogni cristiano sa: l'uomo ha per sempre perduto i "beni preternaturali" che gli erano stati concessi alla creazione. Quello del paradiso terrestre non è un regno come gli altri: nessuno, come Dante sembra lasciar intendere, lo attraversa prima di precipitare nel silenzio divino, nessuno salvo i poeti che da sempre ne sognano. 
La foresta è un non-luogo accedere al quale significa smarrirsi: "non potea rivedere ond'io m’intrassi" (․ 24). Lsssa è "divina" (28, 2), cioè situata nell'impensabile delle origini, ma è anche "antica" (v. 23), cioè luogo che continua a dirsi, dai poeti, nel tempo della parola umana. Luogo poetico per eccellenza, e cioè conosciuto solo attraverso il sogno dei poeti, la foresta primordiale giace tuttaria al di cua della parola, "prima" che essa si dispieghi nella sua interminabile storia. E infatti la colonna sonora di questa scena edenica è piuttosto il canto che la parola: il canto, "in piena letizia" (r. 16) degli uccelli e fin delle foglie "che tenevan bordone a le sue rime" (v. 18); il canto della donna, che "viene" allo smagato viandante "co" suoi intendimenti" (v. 60), intendimenti che il poeta non mostra alcuna intenzione di voler chiarire. La lunga glossa che alla fine spezza l'incanto serve ad ancorare in qualche modo il sogno del poeta a quella doxa che sola lo può rendere comprensibile, lasciando tuttavia nell'ombra "spessa e viva" del sogno il significato della persona, la donna, a cui la roce discettante non sembra del tutto appartenere. Il fantasma femminile che occupa la scena del sogno resta infatti nell'ombra, in perfetta coerenza con il suo significato poetico: solo nel canto conclusiro del Purgatorio la bella innominata, o piuttosto tutta l'operazione simbolica che essa rappresenta, verrà abbreviata in un insolvibile "Matelda": essa è il sogno di Dante, rappresenta l'utopia dell'eros, il momento in cui ta carne, decadendo, si radica nel divino. La donna è visione di sogno, di un sogno intimo e universale insieme, fantasma che appare (cf. "apparve", v. 37) all'uomo-poeta che, per così dire, entra nel sogno da lui stesso fabbricato, "sì come'elli appare/ subitamente cosa che disvia/ per maraviglia tutto altro pensare" (rv. 37 39). Sogno antico, che qui si racconta sulla trasparente falsariga della poesia erotico-cortese, quella stessa poesia che tanti vorrebbero veder definitivamente superata dal poeta, e di cui qui riaffiorano prepotentemente due testi in modo diverso fondanti, il Roman de la Rose e la "pastorella" di Cavalcanti-.

La metafora "paradisiaca" che Guillaume de Lorris aveva forgiato per rappresentare la valenza metafisica del piacere amoroso viene ripresa $\mathrm{e}$ lungamente commentata da Jean de Meun:

Il vit, ce dist, seur l'erbe fresche,

Deduiz qui demenoit sa tresche

Et ses genz o lui querolanz

Seur les florets bien olanz;

Et vit, ce dist li damoisiaus,

Herbes, arbres, bestes, oisiaus:

Et ruisselez et fonteneles 
Bruire et fremir par les graveles,

Et la fontaine souz le pin; ${ }^{8}$

La metafora prescelta da Guillaume è insufficiente, anzi, sbagliata, afferma Jean: le bellezze del vergier non sono che "truffles et fanfellues" (v. 20356). Piacere ha visto "queroles qui faillurent", danze venir meno ( $\mathrm{v}$. 20359), come verranno meno tutte le altre bellezze del giardino. Infatti "Atropos, qui rien ne refuse/ par darriers touz les espiot,/ Fors les dieus, se nul en $i$ ot, / car, sans faille, choses devines/ ne sont pas a la mort enclines". Anche la cristallina luminosità della fontana di Narciso, nella quale il sole fa apparire "colors plus de cent", "jaunes, ynde, vermeil" (rv. 20343-5), è ingannevole: le acque del giardino di Piacere sono in realtà, "pour l'ocurté qui les onnuble" (v. 20458), così scure e torbide "qu'il ne pueent par euls souffire/ A celui qui laienz se mire/ Quant leur clarté d'ailleurs acquierent", che non possono bastare, da sole, a colui che vi si specchia: la loro chiarità viene infatti da altrove (vv. 20458-61). Ecco allora che "corretta" metafora della centralità salvifica di Amore diventa il "parco dell'Agnello", che si trova dentro il giardino del mondo: l'invisibile centro che sta dietro il velo dei sensi, rispetto al quale non solo l'amor cortese, ma tutte le cose del mondo e il mondo stesso non sono che apparenza:

Et qui voudroit au droit aler,

N'en sai ge poprement paler,

Que nus cuers ne porroit penser

Ne bouche d'oume recenser

Les granz biautez, les granz values

Des Choses laienz contenues,

Ne les biaus lieus ne les granz joies

Et pardurables et veroies

Que li queroleeur demainent

Que dedenz la pourprise mainent. ${ }^{10}$

È vero che le gioie del nuovo paradiso di Jean finiscono con lo stranamente somigliare alle stilizzate ingenuità di Guillaume:

Ainz irois par joliveté

Chantant en pardurableté

Motez, conduis et chanconnetes

Par l'erbe verte sor les floretes,

Sous l'olivete querolant. ${ }^{11}$ 
$1 E$ infatti quello di Jean resta inequirocabilmente, nonché contradditoriamente, paradiso della carne, celebrazione di un amore tutto naturale, fatto di piacere e inteso alla procreazione: "Arez, pour Dieu, baron, arez,/ et vos lignages reparez!" (vi: 19705-6). Tutti sappiamo dove termina la queste del pellegrino. Jean de Meun vuole riconquistare lo spazio terrestre che l'escatologia cristiana aveva abbreviato fin quasi ad annullarlo, e infatti per lui "non esistono due regni equipollenti: Amore e Dio; il primo regolatore delle cose quaggiù e l'altro del mondo lassù. Il regno dell'autenticità e verità è uno solo: quello di Dio; e ha sede in un luogo eterno, il Parco/Paradiso" (Picone, 51). Per questo, e nonostante l'apparente escatologia, la sua indagine resta conclusa entro i limiti dell'esperienza mondana, non guarda oltre la morte, non cerca la sintesi filosofico-teologica così accanitamente perseguita da Dante: che infatti unifica il vergier di Guillaume e il parc di Jean in un solo paradiso, terrestre ma inattingibile, u-topia assoluta nella quale tuttavia "rimane detto" quello che l'amore carnale è, un effimero che dice l'eterno. Tuffandosi, o piuttosto lasciandosi immergere nell'acqua "bruna bruna" eppure limpida — "che nulla nasconde"- che bagna quel "mondo sanza gente" mai raggiunto da Ulisse, l'uomo "dimentica" il male e "ricorda" il bene. Come dio.

Se il Roman de la Rose appare solo come in filigrana, la ballata del secondo e maggiore Guido sottende il testo dantesco in modo preciso e immediato, e testimonia ben più che un omaggio nei confronti dell'amico "tradito". In un boschetto trora" pasturella è un componimento erotico, un felice hapax di forma e di sentimento nella produzione di un poeta che nelle cose d'amore è notoriamente più portato, piuttosto che al riso, alle lacrime e al lutto della memoria. Non diversamente, in questo, dall'amico, per il quale la "pace" desiderata dal corpo e promessa dagli occhi fatica a trovarsi raffigurata, e, se lo è, lo è quasi parenteticamente, istante di suprema grazia e bellezza incalzato dall'angoscia: "ché 'l mal d'amor non è pesante il sesto/ ver' ch'è dolce lo ben" (Com piì ri fere Amor, 7-8); "pensando a quel che d'Amore ho provato, / l'anima mia non chiede altro diletto" (I o doloroso amor, 29-30); "ché se 'l martiro è dolce,/ la morte de' passare ogni altro dolce" (Io son venuto, 64-65). O invano anticipato dal desiderio:

\footnotetext{
"Noi darem pace al core, a voi diletto", diceano a li occhi miej quei de la bella donna alcuna volta; ma poi che sepper di loro intelletto che per forza di lei m'era la mente già ben tutta tolta,
} 
con le insegne d'Amor dieder la volta,

si che la lor vittoriosa vista

poi non si vide pur una fiata:

ond'è rimasa trista

l'anima mia che n'attendea conforto,

e ora quasi morto

rede lo core a cui era sposata

e partir la convene innamorata.

(È m’incresce di me si duramente, 15-28)

Per non parlare di quella "pace" violenta che il poeta vorrebbe "rendere", "con amore", alla "scherana micidiale e latra" che lo tormenta (Così nel mio parlar voglio esser aspro, 58, 78). Se l'esperienza erotica si presenta ai due amici prevalentemente come tormento e "morte", Guido sa almeno una volta esprimerne senza mezzi termini la gioia:

Per man mi prese, d'amorosa roglia, e disse che donato m'avea 'l core; menommi sotto una freschetta foglia, là dor'i' vidi fior' d'ogni colore; e tanto vi sentio gioia e dolzore che 'l die d'Amore - mi parea vedere.

Ecco l" "incantamento" da condividere con Guido: il "talento" dello "stare insieme", e insieme con donne: "e ciascuna di lor fosse contenta/ sì

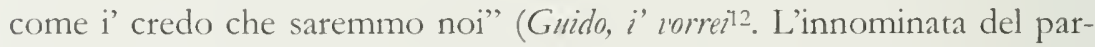
adiso terrestre dantesco, questa lieve gentile figura del piacere d'amore, risponde, nel "sogno" della poesia, alla richiesta del poeta che vorrebbe avere "nova donna" "in un bel prato d'erba,/ innamorata com'anco fu donna" (Alpoco giorno, 7; 28-30), e risponde proprio, nell'incanto del sogno, "come donna innamorata" (Purg. 29, 1).

L'innominata è "soletta" (28, 40; cf. 31,92$)$, e il suo contegno, come di donna disponibile che si "scaldi" "a' raggi d'amore" (v: 43), fa pensare alla madonna Oziosa del Fiore (79): canta e sceglie "fior da fiore" (v. 41). I suoi "sembianti, che soglion esser testimon del core" (vv: 44-45) lo confermano. Allo sguardo del protagonista, che l'accarezza di sotto in su, dai piedi agli occhi bassi, risponde finalemente come Venere, da "sotto le ciglia", col "dono" de "li occhi suoi" (v. 63, 66). E sorride: "Ella ridea da l'altra riva dritta,/ trattando più color con le sue mani,/ che l'alta terra sanza seme gitta" (vr. 67-69). Anche le similitudini, tutte di natura erotica, da Proserpina a Venere a Ero e Leandro, non lasciano dubbi su quale sia la natura del luogo poetico in cui ci troviamo. Si dirà, si è detto, che, diver- 
samente dalla ballata di Cavalcanti, qui non si dà consumazione. Non occorre insistere che una rappresentazione "tecnica", evitata del resto anche da Cavalcanti, risulterebbe in un disastro poetico e filosofico: nel fitto di una rigorosissima meditazione intesa a drammatizzare la disappartenenza di eros alla storia, come a dire la trascendenza assoluta dell'amore, un Durante alla conquista del fiore introdurrebbe una grottesca incongruenza. Eppure una rappresentazione, a saperla intendere, Dante la dà: "la donna ch'io avea trovata sola/ sopra me vidi, e dicea "Tiemmi, tiemmi!"” (Purg. 31. 92-93). E cosi "la bella donna ne le braccia aprissi;/ abbracciommi la testa e mi sommerse" (vт: 100-101); "indi mi tolse, e bagnato mofferse..." (v. 103). l.e acque in cui il poeta protagonista si rappresenta sommerso ad opera della bella donna, insieme con "lo dolce ber che mai m'arria sazio" (Purg. 33, 138) a cui la stessa lo induce, dicono il bagliore del felice oblio, là dove la "conoscenza del bene e del male" si annulla in quel "fuori dal tempo" che è il divino istante dell'amore umano. L'essenziale risulta detto nell'unico modo in cui potrebbe esserlo senza intaccare la coerenza poetica di un luogo che ruole esplicitamente essere utopia.

La donna ride, e il suo terrenissimo riso si rimarca nella risposta al parentato "sospetto" in cui qualcuno (forse "l'annoiosa gente"?) potrebbe cadere: ma come? Dante ci parla di "questo" riso in un luogo cosi santo? Ed ecco la risposta "che puote disnebbiar vostro intelletto" (v. 81): il luogo è, per l'appunto, stato "eletto a l'umana natura per suo nido" (v. 78), e su questo, sulla misteriosa "originaria" sacralità della carne, "luce rende il salmo Delectasti" (v. 80). l commentatori riferiscono generalmente questa citazione a un versetto del salmo 91, non senza esprimere qualche perplessità nei confronti di una trascuratezza dantesca che lo arrebbe indebitamente trasformato in titolo: ma allora, una volta comunque ammessa la licenza poetica, è almeno ipotizzabile che il riferimento sia invece a quel salmo 36 che san Tommaso cita proprio nel suo discorso sul rapporto tra ragione e piacere: "videtur quod non omnis delectatio sit mala". Non siamo tornati né ad Agostino — di "ragione" nell'eden dantesco, giustamente, non c’è traccia- né a Jean de Meun, ché davvero qui non si tratta di una celebrazione della bontà della natura. Siamo invece nel vasello di Guido, nel suo boschetto amoroso: che però è situato fuori della storia, nell'istante u-topico della sempre perduta origine. la "pace" dell'amore non è "più" "etterna", e cioè perennemente godibile lungo la linea del tempo: per la "difalta" (v: 94) dell'uomo, la dimora dell'amore è ormai aleatoria, intermittente, istantanca. La "difalta" dell'uomo è, precisamente, il suo "fallere", la sua caduta nella socictà e nella storia, là dore "onesto riso e dolce gioco" si cambiano "in pianto e affanno" (v. 95). Il riso di 
Matelda appartiene all'u-topia; il riso di Francesca è della storia, "del viver ch'è un correre a la morte" (Purg. 33, 54). L'amore è "impossibile" nella storia, non appartiene ad essa: in questo il doloroso Guido ha ragione. Ciò che il suo amico vede, e può darlo guardando nel "miraglio" di Rachele che solo a pochi è offerto, è che il riso dell'amore carnale è tutt'altro che privo di spessore teologico. Nell'oblio della ragione che caratterizza l'amore, la "tramortita virtù" dell'uomo viene ravvivata (cf. Purg. 33, 129); terrenamente amando, l'uomo trapassa, momentaneamente e quodammodo' ${ }^{12}$, in dio.

La liturgia erotica, o piuttosto l'eros liturgico che vediamo compiersi nel paradiso terrestre non rappresente il "ritorno" a un felice stato di giustizia primitiva. Come dice Aristotele seguito da san Tommaso, "iustitia est virtus ad alterum" (citato da Boyde, 202), la giustizia è ideale della società, e qui non siamo nella società, siamo in "originaria", perfetta, solitudine. Non si dà ritorno alla società, l'unico ritorno possibile è alla morte. Tra il poeta e il suo simbolo, fuori delle mura fiammanti del mondo, si svolge un rito in cui eros e morte si sovrappongono in una dimenticanza senza ritorni. La conoscenza del bene e del male, travalicando i limiti del pensiero, diventa innocenza divina: "eritis sicut dii". Se Atropos risparmia gli dei, è perché sta dalla loro parte. Nel frattempo della storia, della ragione e della parola, amore detta, e l'uomo, senza fine disnebbiandosi, scrive.

\section{Emory University}

\section{NOTE}

${ }^{I}$ Fenzi, alla cui osservazione sui versi di Purg. $31 \mathrm{mi}$ ispiro, propone per altro di sostituire Anovità" con Avanità", adducendo l'uso che del secondo termine Dante fa nel libello a proposito della Adonna pietosa". Non mi pare che quest'uso sia dirimente, specialmente se nel Comivio lo stesso amore è piuttosto connotato come Anovità", $e$ in ogni caso lo stesso Fenzi finisce con l'assimilare Anovità" a Avanità".

2 Cf. Summa, I lxxix 8: Aratio et intellectus in homine non possunt esse diversae potentiae. Quod manifeste cognoscitur, si utriusque actus consideretur. Intelligere enim est simpliciter veritatem intelligibilem apprehendere. Ratiocinari autem est procedere de uno intellecto ad aliud, ad veritatem intelligibilem cognoscendam.... ratiocinari comparatur ad intelligere sicut moveri ad quiescere, vel acquirere ad habere, quorum unum est perfecti, aliud autem imperfecti".

3 Sorprende trovare nel commento di Foster e Boyde (II, 299) una lunga considerazione sul significato da attribuire a questi versi, con preferenza dichiarata per una lettura che vi vedrebbe raccomandato un Aactive self-disfigurement".

4 Questo mi pare il senso di "quamris sit in eo quod convenit rationi".

5 Il verso 133 , "pria ch'i' scendessi a l'infernale ambascia" pare riferirsi let- 
teralmente alla morte di Adamo, non alla scacciata dal Paradiso. Ma la brevita della lingua "adamitica" è comunque riferita a un tempo altrettanto miticamente breve, all'impensabile della pre-storia.

6 Le precise corrispondenze testuali tra la ballata di Caralcanti "in un boschetto trova' pasturella", e l'episodio della "bella donna" nel Purgatorio sono state particolareggiatamente descritte da Teodolinda Barolini (1984, 149-153). Ad esse si potrebbe aggiungere il particolare dei piedi: la pasturella c̀ "scalza", la donna edenica è paragonata a "donna che balli,/ e piede innanzi piede a terra mette" (เт: 52-54).

7 Vr. 20341-49. "L'amante dice di arer risto sull'erba fresca Piacere ballare la sua tresca, e la sua gente ballare con lui sui fiori aulenti. E il giovane dice di aver vito erbe, alberi, animali, uccelli; e ruscelli e sorgenti mormoranti e frementi tra i sassi; e la sorgente sotto il pino" (le traduzioni sono mie).

8 V v. 20367-72. "Atropos, che non rifiuta nulla, alle loro spalle li spia tutti tranne gli dei, se ce ne sono, poiché sicuramente le cose divine non sono soggette alla morte".

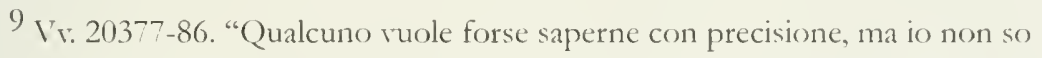
parlarne con proprietà, poiché nessun cuore potrebbe concepire, né bocca d'uomo dire, le grandi bellezze, il grande valore delle cose che ri sono contenute, né i bei luoghi né le grandi gioie durature e vere di cui godono le persone danzanti che abitano in questo recinto" .

10 "Invece ve ne andrete in allegria, perennemente cantando mottetti, cori e canzonette tra l'erba verde e i fiorellini, danzando sotto l'ulivo" (rv. 20659-20663). L'ulivo sostituisce in senso che si vorrebbe cristiano il pino di Guillaume.

12 Ma si pensa anche al sonetto L'na ricca rocca e forte manto, dell'altro amico, Cino:

Una ricca rocca e forte manto

volesse Dio che monte ricco avesse,

che di gente nemica non temesse,

avendo un'alta torre ad ogni canto;

e fosse d'ogni ben compita quanto

core pensare e lingua dir potesse,

e quine poi lo dio d'amore stesse

con li amorosi cori in gioia e canto.

E poi rorrei che nel mezzo surgesse

un'acqua rertudiosa d'amor tanto,

che lor bagnando dolce vita desse;

e perché più fedele il meo cor vanto,

vorrei che 'l gonfalon tra quei tenesse

che portan di soffrir pietoso manto.

13 Cf. il vertiginoso "quodammodo" di Mon. III xv 17: "cum mortalis ista felicitas quodammodo ad immortalem felicitatem ordinetur". 


\section{OPERE CITATE}

Barolini, Teodolinda. Dante's Poets. Princeton: Princeton University Press, 1984. "Dante and Cavalcanti (On Making Distinctions in Matters of Love)". Dante Studies 116 (1998). 31-63

Andrea Cappellano, Trattato d'amore. Testo latino del sec. XII con due traduzioni toscane inedite del sec. XIV', a cura di S. Battaglia, Roma: Perrella, 1947

Boyde, Patrick. Human I 'ices and Human W"orth in Dante's Comedy. Cambridge, UK ; New York, NY, LSA : Cambridge University Press, 2000.

Chiavacci Leonardi, Anna Maria (con il commento di). Commedia, 3 voll. Milano: Mondadori, 1991.

Dartmouth Dante Project, Inferno.

De Robertis, Domenico. ASulla cultura giovanile di Dante". in Letture classensi 4, 1973. 231-260.

Enciclopedia dantesca, Roma: Istituto della Enciclopedia ltaliana, 1971 (4: 831-841). Fenzi, Enrico. " > Costanzia de la ragione' e >malvagio desiderio" ( I .N., XXXIX, 2): Dante e la donna pietosa". La gloriosa doma de la mente: a commentary on the I'ita Nuora, ed. V'incent Moleta, Firenze: Olschki, 1994. 195-224.

Foster, Kenelm and Boyde, Patrick, eds. Dante's Lyric Poetry. Oxford: Oxford University Press, 1967.

Le Roman de la Rose. "Edition d'après les manuscrits BN 12786 et BN 378. Traduction, présentation et notes par Armand Strubel. Paris : Librairie Générale Française, 1992.

Picone, Michelangelo. "T ita nuova" e tradizione romanza. Padova: Liviana, 1979. 